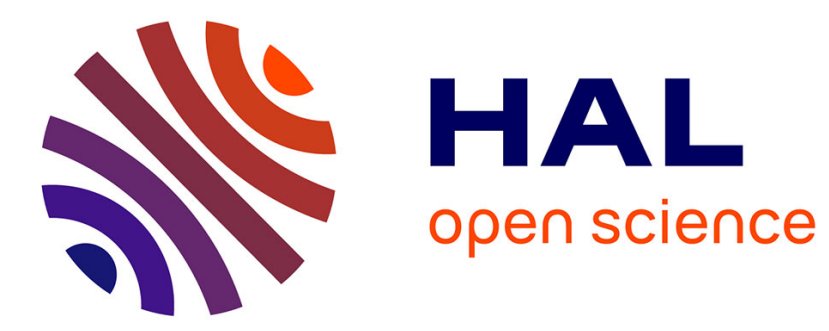

\title{
Local sensitivity for uncertainty analysis in one-dimensional open channel flow modelling
}

Carole Delenne, Vincent Guinot, Bernard Cappelaere

\section{To cite this version:}

Carole Delenne, Vincent Guinot, Bernard Cappelaere. Local sensitivity for uncertainty analysis in one-dimensional open channel flow modelling. La Houille Blanche - Revue internationale de l'eau, 2013, 1, pp.50-59. 10.1051/lhb/2013008 . hal-01196828

\section{HAL Id: hal-01196828 \\ https://hal.science/hal-01196828}

Submitted on 14 Sep 2015

HAL is a multi-disciplinary open access archive for the deposit and dissemination of scientific research documents, whether they are published or not. The documents may come from teaching and research institutions in France or abroad, or from public or private research centers.
L'archive ouverte pluridisciplinaire HAL, est destinée au dépôt et à la diffusion de documents scientifiques de niveau recherche, publiés ou non, émanant des établissements d'enseignement et de recherche français ou étrangers, des laboratoires publics ou privés. 


\title{
LOCAL SENSITIVITY FOR UNCERTAINTY ANALYSIS IN ONE-DIMENSIONAL OPEN CHANNEL FLOW MODELLING
}

\author{
Carole DELENNE ${ }^{1}$, Vincent GUINOT \\ Université Montpellier 2, HydroSciences Montpellier (UMR 5569) \\ carole.delenne@univ-montp2.fr; Vincent.guinot@univ-montp2.fr \\ Bernard CAPPELAERE \\ IRD, HydroSciences Montpellier (UMR 5569) \\ bernard.cappelaere@univ-montp2.fr
}

Uncertainty analysis may be of high importance in the field of water engineering. Sampling-based techniques, that resort to extensive exploration of the space of possible model inputs are a common approach. When inputs uncertainties can be described statistically by means of probability distribution functions, Monte Carlo simulation yields a statistical description of uncertain model outputs. However, the combination of commonly encountered input space dimensions and model CPU times often makes this approach hardly practicable. We propose to assess the potential of a local sensitivity analysis for uncertainty analysis in the modelling of open channel flows with the one-dimensional shallow water equations, which are generally viewed as being largely non linear. The first-order local sensitivity is simply assessed using the so-called empirical method requiring only two runs of the model (for a single uncertain parameter). The global sampling-based approach consists in running Monte Carlo simulations with the hydrodynamic model, given a statistical distribution of the model input parameters. Monte Carlo results can be analysed through classical statistical estimators, such as mean and standard deviation. It is shown that these characteristics can also be accurately estimated from the local sensitivity and the input distribution. Steady state and highly transient configurations (gate opening and closing) are explored, accounting for uncertainty of the main parameters (bottom slope, friction coefficient, boundary and initial conditions).

\section{Key words:}

Monte Carlo; computation time; variance based approach; backwater curve; gate closing

\section{Sensibilité locale pour l'analyse d'incertitude dans la modélisation 1D des écoulements à surface libre en canal}

L'analyse d'incertitude joue un rôle important dans les domaines de l'ingénierie de l'eau. Les techniques classiques sont basées sur l'échantillonnage et l'exploration extensive de l'espace des paramètres du modèle. Lorsque l'incertitude des paramètres peut être décrite statistiquement par des fonctions de distribution de probabilité, l'approche Monte Carlo permet d'obtenir une description statistique des résultats du modèle. Cependant, les dimensions généralement rencontrées pour l'espace des paramètres associées au temps CPU de simulation des modèles rendent souvent cette approche difficilement utilisable. On propose d'évaluer les potentiels d'une analyse de sensibilité locale pour l'analyse d'incertitude, dans le cas de la modélisation à surface libre dans un cannal par les équations "shallow water" $1 \mathrm{D}$, qui sont généralement considérées comme fortement non linéaires. La sensibilité locale de premier ordre est simplement calculée par la méthode dite empirique, qui ne nécessite que deux simulations du modèle (lorsqu'un seul paramètre est incertain). L'approche globale, basée sur un échantillonnage de l'espace des paramètres, consiste à générer des simulations de Monte Carlo avec le modèle hydrodynamique, à partir

\footnotetext{
${ }^{1}$ corresponding author
} 
d'une distribution statistique des paramètres d'entrée. Les résultats des simulations de Monte Carlo peuvent être analysés à travers des estimateurs statistiques classiques comme la moyenne ou l'écart-type. On montre que ces caractéristiques peuvent également être estimées à partir de la sensibilité locale et de la distribution du paramètre d'entrée. Des configurations en régime permanent ou hautement transitoires (ouverture et fermeture de vannes) sont explorées, avec prise en compte de l'incertitude sur les principaux paramètres (pente du fond, coefficient de frottement, conditions initiales et aux limites).

\section{Mots-Clés:}

Monte Carlo; temps de calcul; approche type variance; courbe de remous; fermeture de vanne

\section{INTRODUCTION}

Sensitivity analysis methods can be classified into three main categories [Saltelli et al., 2000]: screening, global and local approaches. The first one requires low computational effort but gives only qualitative sensitivity measures, and will not be considered in this paper (see [Saltelli et al., 2000] for more details). The global approach is based on exploring the whole space of the input parameters. Given a description of their uncertainty through probability distribution functions, the uncertainty in the model output can be estimated after a high number of simulations. The advantage of Monte Carlo analysis is that it can be used for nonlinear models, as complex as they can be, with as many input and output variables as needed. However, the more complex the model, the more costly the computation for the simulations and post-processing. Contrarily to the global approach, local methods estimate the effect of a small variation in the input parameters around nominal values. The local sensitivity is usually defined as the partial derivative of the output variable with respect to a given parameter. This derivative can be assessed either based on model equations or simulation outputs. In the first case, the direct approach consists in first differentiating the model equations and then, solving the resulting sensitivity equations. In the second case, the classical empirical (or finite difference) approach consists in performing two simulations with two slightly different values of the parameter of interest and then differentiate the output by computing the difference between both results, normalised by the parameter variation. Contrarily to the global approach, local methods thus require only one or two simulations. As shown in e.g. [Delenne et al., 2011; Guinot et al., 2009a] the first, direct approach can deal with discontinuous flow solutions. However the second has the advantage of being straightforward for any kind of model even of "black box" type and will be used in this paper for the sake of simplicity.

Uncertainty and sensitivity analysis are of high importance in the fields of water engineering and management such as resource allocation, water quality, hazards (flood, tidal wave or dam/dike break) or impacts of climate change on hydrological systems (see e.g. [Hall et al., 2005; Pappenberger et al., 2006; Delenne et al., 2008; Kay and Davies, 2008; Minville et al., 2008; Roux and Dartus, 2008]). For instance, uncertainty arising from model calibration is increasingly handled using the empirical, sampling-based Generalised Likelihood Uncertainty Evaluation (GLUE) methodology, developed in the hydrological modelling context [Beven and Freer, 2001]. Sensitivity analysis has also been identified as being of great importance in the field of irrigation channel management (see e.g. [Malaterre et al., 2011]).

The aim of this paper is to compare the local, empirical method to the classical global Monte Carlo analysis for uncertainty analysis in the field of irrigation channel management. For the sake of simplicity, the comparison is performed with the 1D Shallow water equations. The structure of the paper is as follows. In section 2, the governing equations are recalled as well as the numerical method used to solve them. Section 3 gives the framework of sensitivity and uncertainty analysis. Section 4 presents results obtained for a steady-state application (single-parameter analysis and simultaneous variation of several parameters, either independently or with a non-zero correlation) and two highly transient test cases: the sudden opening or closing of a gate, with an uncertainty analysis with respect to different parameters (friction coefficient, initial water depth, initial flow velocity...) 


\section{GOVERNING EQUATIONS}

\subsection{One-dimensional Shallow Water Equations}

The one-dimensional shallow water equations can be written in vector form as

$$
\frac{\partial \mathbf{U}}{\partial t}+\frac{\partial \mathbf{F}}{\partial x}=\mathbf{S} \text { with } \mathbf{U}=\left(\begin{array}{c}
h \\
q
\end{array}\right), \mathbf{F}=\left(\begin{array}{c}
q \\
q^{2} / h+g h^{2} / 2
\end{array}\right), \mathbf{S}=\left(\begin{array}{c}
0 \\
g h\left(S_{0}-S_{f}\right)
\end{array}\right)
$$

where $h$ is the water depth, $q$ is the unit-discharge, $g$ is the gravitational acceleration, $S_{0}=-\partial z_{b} / \partial x$ is the bottom slope (with $z_{b}$ the bottom elevation) and $S_{f}$ is the friction slope computed using a classical Manning law under the wide channel approximation:

$$
S_{f}=q|q| n_{M}^{2} h^{-10 / 3}
$$

where $n_{M}$ is the Manning's friction coefficient.

\subsection{Numerical method}

The flow equation (1) is discretized using a classical explicit finite volume formulation:

$$
\mathbf{U}_{i}^{n+1}=\mathbf{U}_{i}^{n}-\frac{\Delta t}{\Delta x_{i}}\left(\mathbf{F}_{i-1 / 2}^{n+1 / 2}-\mathbf{F}_{i+1 / 2}^{n+1 / 2}\right)+\Delta t \mathbf{S}_{i}^{n+1 / 2}
$$

where $\Delta t$ is the computational time step, $\Delta x_{i}$ is the width of the cell $i, \mathbf{U}_{i}^{n}$ is the average value of $\mathbf{U}$ over the cell $i$ at the time level $n, \mathbf{F}_{i-1 / 2}^{n+1 / 2}$ is the average value of the flux $\mathbf{F}$ through the interface $i-1 / 2$ (between the cells $i-1$ and $i$ ) between the time levels $n$ and $n+1, \mathbf{S}_{i}^{n+1 / 2}$ is the average value of $\mathbf{S}$ over the cell $i$ between the time $n$ and $n+1$.

The flux $\mathbf{F}$ is computed for each interface from the solution of a Riemann problem defined at the cell interface. Each Riemann problem is solved using an approximate Riemann solver based on the HLL/HLLC formalism [Harten et al., 1983; Toro et al., 1994]. In the proposed discretization, an explicit method is used, i.e. the flux $\mathbf{F}$ and the source term are computed from the known solution at the time level $n$. The two waves separating the intermediate region of constant state from the left and right states of the Riemann problem are assumed to be discontinuities, having respectively the following celerities: $\left(\lambda^{-} ; \lambda^{+}\right)=(u-c ; u+c)$. The source term $S$ in (1) is split into two parts with $-\lambda^{-} /\left(\lambda^{+}-\lambda^{-}\right)$and $\lambda^{+} /\left(\lambda^{+}-\lambda^{-}\right)$as respective weights for the contributions of an interface to its right and left cells (see e.g. [Guinot et al., 2009a] for a detailed description of the numerical method).

\section{SENSITIVITY AND UNCERTAINTY ANALYSIS}

\subsection{Monte Carlo simulation for global estimation of model uncertainty}

Parameter uncertainty is modelled using a Beta distribution, which has the following probability density function

$$
f(\phi ; \alpha, \beta)=\frac{\phi^{\alpha-1}(1-\phi)^{\beta-1}}{\int_{0}^{1} u^{\alpha-1}(1-u)^{\beta-1} \mathrm{~d} u}
$$

where $\phi \in[0,1]$ and $(\alpha, \beta)$ are shape parameters taken here as $\alpha=\beta=5$ (symmetric distribution). Unlike the widely used Gaussian distribution, this function has a finite support, thereby avoiding possible non-physical values of the parameters (such as negative values for the friction coefficient). The distribution obtained for $\phi$ is then centred onto the nominal value $\psi^{0}$ of the parameter of interest $\psi$ and rescaled to a min-max interval: $\left[\psi^{0}-\Delta \psi ; \psi^{0}+\Delta \psi\right]$. Monte Carlo simulations are performed using a sample of the parameter $\psi$ generated by a random sample of size $N$ drawn from a Beta distribution.

When several parameters are considered uncertain simultaneously, they are either taken independent or correlated. Model uncertainty is assessed for the global approach as the sample mean $\left(\mu_{\mathbf{U}}(x)\right)_{G}$ and standard deviation $\left(\sigma_{\mathbf{U}}(x)\right)_{G}$ of the $N$ model outputs $\mathbf{U}(x)_{n}, n=1 \ldots N$. 


\subsection{First order, local sensitivity for estimation of model uncertainty}

The local sensitivity of a variable $\mathbf{U}$ with respect to any parameter $\psi$ is defined as the partial derivative of $\mathbf{U}$ with respect to $\psi$

$$
\mathbf{s} \equiv \frac{\partial \mathbf{U}}{\partial \psi}=\left(\begin{array}{c}
\eta \\
\theta
\end{array}\right)
$$

For the sake of simplicity, the local sensitivity is not computed by solving sensitivity equations such as presented e.g. in [Delenne et al., 2011; Guinot and Delenne, 2012; Guinot et al., 2009b] in the case of discontinuous flows, but using the so-called empirical approach. This finite difference approach consists in performing two simulations with two slightly different values of the parameter of interest and computing the difference between both results, normalised by the parameter variation. The local sensitivity of the variable $\mathbf{U}$ with respect to a given parameter $\psi$ around its nominal value $\psi_{0}$ is thus defined as:

$$
\mathbf{s}_{\psi_{0}} \equiv\left(\begin{array}{c}
\eta \\
\theta
\end{array}\right)=\frac{\mathbf{U}\left(\psi_{0}+\Delta \psi\right)-\mathbf{U}\left(\psi_{0}\right)}{\Delta \psi}
$$

where $\Delta \psi$ is a small variation in the parameter.

The model output produced with the mean, i.e. nominal parameter set provides an estimation with first-order accuracy of the output mean, denoted $\left(\mu_{\mathbf{U}}(x)\right)_{L}$. In the case when one parameter $\psi$ is allowed to vary at a time, the standard deviation $\sigma_{\mathbf{U}}(x)$ of the model output at each abscissa $x$ can be first-order estimated from the sensitivity solution obtained with the nominal parameter set, as $\left(\sigma_{\mathbf{U}}\right)_{L}=|\mathbf{s}| \sigma_{\psi}$, i.e. for the output $h$ :

$$
\left(\sigma_{h}\right)_{L}=\left|\eta_{\psi}\right| \sigma_{\psi}
$$

where $\sigma_{\psi}$ is the standard deviation of the input distribution of the parameter $\psi$.

In the case when $N$ parameters vary at the same time, with possible correlation between parameters, the firstorder estimate of standard deviation writes for output $h$ (the same equation holds for $q$ with $\theta_{\psi_{i}}$ in place of $\eta_{\psi_{i}}$ ):

$$
\left(\sigma_{h}\right)_{L}=\left(\sum_{i=1}^{N} \eta_{\psi_{i}}^{2} \sigma_{\psi_{i}}^{2}+\sum_{i \neq j, i j=1}^{N} \operatorname{cov}\left(\psi_{i}, \psi_{j}\right) \eta_{\psi_{i}} \eta_{\psi_{j}}\right)^{1 / 2}
$$

where $\eta_{\psi_{i}}$ is the sensitivity of $h$ with respect to parameter $\psi_{i}, \sigma_{\psi_{i}}^{2}$ is the variance of the input distribution for the parameter $\psi_{i}, \operatorname{cov}\left(\psi_{i}, \psi_{j}\right)$ is the covariance between the input distribution of parameters $\psi_{i}$ and $\psi_{j}$. For the sake of conciseness, equation (7) is given for two by two correlated parameters, but the formulation would be valid for cross-correlation between more than two parameters.

The two methods are compared through their estimations of the model output moments, $\mu$ and $\sigma$. Taking the global estimates $\mu_{G}$ and $\sigma_{G}$ as the reference, average relative errors are computed for each moment as:

$$
\begin{aligned}
& e_{\mu}=\frac{\Delta x}{L} \sum \frac{\left|\mu_{G}-\mu_{L}\right|}{\mu_{G}} \\
& e_{\sigma}=\frac{\Delta x}{L} \sum \frac{\left|\sigma_{G}-\sigma_{L}\right|}{\sigma_{G}}
\end{aligned}
$$

where $\Delta x$ is a spatial discretization step along the length $L$ of river reach, and the summation applies to the resulting space steps. 


\begin{tabular}{lll} 
Symbol & Meaning & Value \\
\hline$g$ & Gravitational acceleration & $9.81 \mathrm{~m} \mathrm{~s}^{-2}$ \\
$L$ & Channel length & $3000 \mathrm{~m}$ \\
$S_{0}^{0}$ & Nominal value of the bottom slope & $10^{-3}$ \\
$q^{0}$ & Nominal value of the prescribed unit discharge & $3 \mathrm{~m}^{2} \mathrm{~s}^{-1}$ \\
$h_{d s}^{0}$ & Nominal value of the downstream water depth & $2 \mathrm{~m}$ \\
$n_{M}^{0}$ & Nominal value of the Manning's friction coefficient & $0.025 \mathrm{~m}^{-1 / 3} \mathrm{~s}$ \\
$\Delta x$ & Discretization cells width & $0.1 \mathrm{~m}$ \\
\hline
\end{tabular}

Table 1: Test 1 - Steady state flow. Parameters of the test case.

\section{TEST CASES: 1D OPEN CHANNEL FLOW APPLICATIONS}

\subsection{Test 1 - Steady flow on a sloping bed (backwater curve)}

The backwater curve is a simplification of one-dimensional shallow water equations (1) in the case of a permanent flow:

$$
\begin{cases}\frac{\mathrm{d} h}{\mathrm{~d} x}= & \frac{S_{0}-S_{f}}{1-\mathrm{Fr}^{2}} \\ h(L)= & h_{d s}\end{cases}
$$

where $\mathrm{Fr}=\sqrt{q^{2} /\left(g h^{3}\right)}$ is the Froude number for a rectangular channel, $L$ is the domain length and $h_{d s}$ is the prescribed water depth at the downstream boundary. The four parameters of this test case are: the bottom slope $S_{0}$, the prescribed unit discharge $q$, the prescribed downstream water depth $h_{d s}$ and the Manning's friction coefficient $n_{M}$. The parameters values are given in Table 1. The unique output variable is the water depth $h(x)$ as a function of space.

\subsubsection{One uncertain parameter}

We first explore the case of one-at-a-time (OAT) uncertainty analysis. A convergence analysis (not presented here for the sake of conciseness) on the number $N$ of Monte Carlo simulations shows that $N=5000$ simulations are required to ensure convergence. For each parameter, $N=5000$ simulations are thus performed, using an input distribution following a Beta law (Eq 4$)$ rescaled in $\psi^{0}(1 \pm 50 \%)$ for the parameter of interest and nominal values for the three other parameters (Figure 1).

The $\mathrm{dX}$ deciles of the output water depth distribution $\left(h_{\mathrm{MC}}^{\mathrm{dX}}\right)$ obtained from theses simulations are plotted in Figure 2 for each parameter and with $\mathrm{dX}=10 \%, 50 \%$ and $90 \%$. These deciles are also estimated from the local sensitivity of each parameter $\psi$ using the following equation:

$$
\left(h_{\mathrm{Local}}^{\mathrm{dX}}\right)_{\psi}=h^{0}+\eta_{\psi}\left(\psi^{0}-\psi^{\mathrm{dX}}\right)
$$

where $h^{0}$ is the result obtained with the nominal values of the four parameters, $\eta_{\psi}$ is the sensitivity of $h$ with respect to the parameter $\psi, \psi^{0}$ is the nominal value of the parameter of interest and $\psi^{\mathrm{dX}}$ is the value of the $\mathrm{dX} \%$ decile of the parameter distribution. It can be seen from Figure 2 that the deciles are well reproduced by the local approach for each parameter, except the $10 \%$ and $90 \%$ deciles when the uncertain parameter is the prescribed downstream water depth. This is due to the fact that the curvature of the output water depth is opposite depending on whether the downstream water depth is higher or lower than the normal height as shown in Figure 3.

To assess the robustness of the local approach to uncertainty analysis, the output mean and standard deviations obtained from the two methods are compared for different possible variations of each parameter. In each case, $N$ simulations are performed, using an input distribution following a Beta law (4) rescaled in $\psi^{0}(1 \pm X \%)$, with $X \in[10 ; 70]$. The standard deviations obtained with local and global methods are compared through the relative errors computed by equations (8) and (9) as a function of $X$ in Figure 4. For the same reason as before, the standard deviation of output water depth is incorrectly estimated by the local sensitivity approach when $h_{d s}$ is the uncertain 

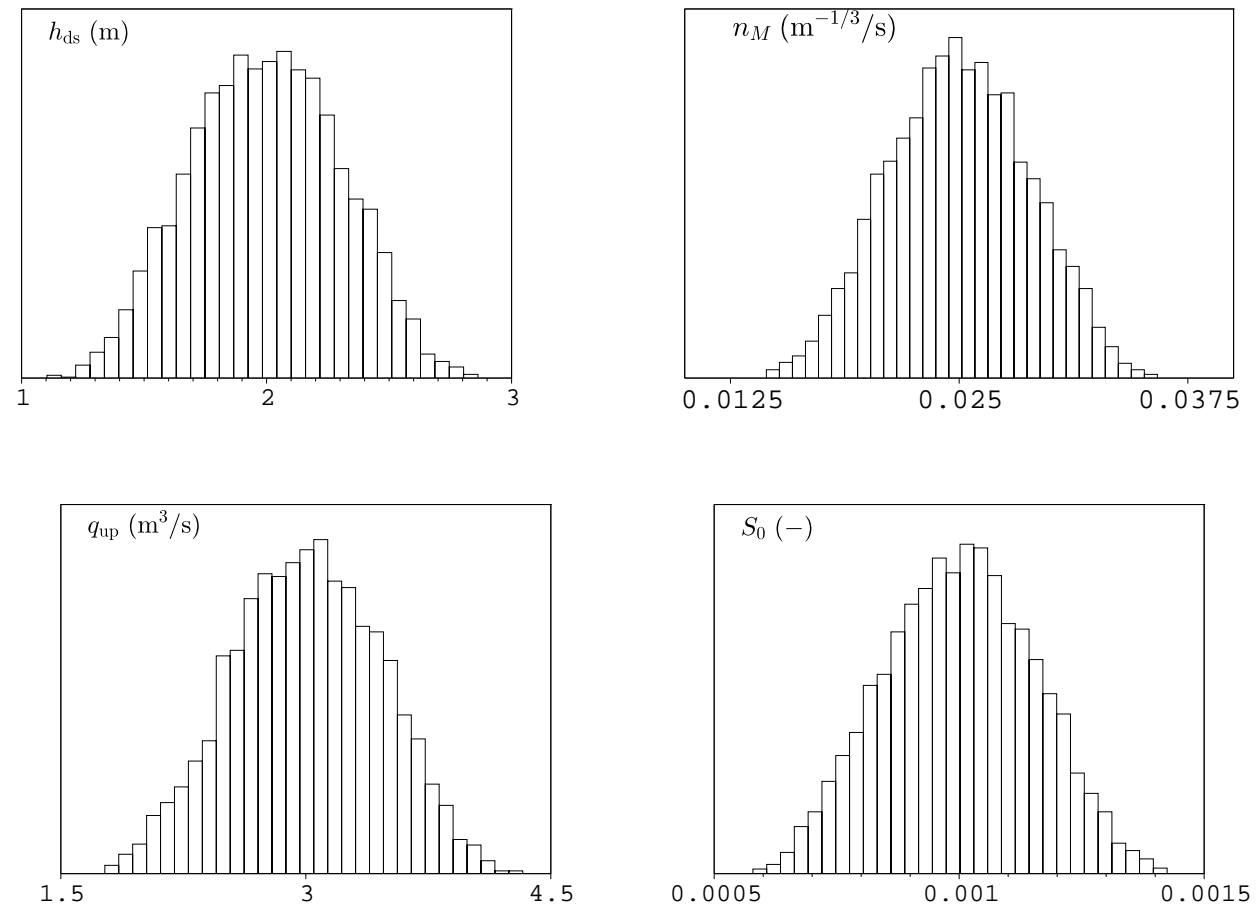

Figure 1: Test 1 - steady state flow. Distributions of the uncertain parameters.

parameter. However, for the other three parameters, the relative difference between the two standard deviation estimates is smaller than $3 \%$ of the global estimates even when the parameters are allowed to vary by as much as $70 \%$. Results are better for the mean estimates with an error lower than $1.5 \%$ even when the uncertain parameter is $h_{d s}$.

This first test case shows that the local sensitivity, that requires only two simulations, can be accurately used to retrieve global results, requiring 5000 runs of the model, in the case of one-at-a-time uncertainty analysis of the backwater curve.

\subsubsection{Two correlated parameters}

This section is devoted to the study of the influence of two simultaneous uncertain parameters, the boundary condition $h_{\mathrm{ds}}$ and the unit-discharge $q$. The number of simulation for the global approach has been set to $N=$ 10000. The prescribed downstream water depth is taken in a Beta law with $h_{\mathrm{ds}} \in[1.5 ; 2.5] \mathrm{m}$ (yielding a standard deviation of $\sigma_{h}-\approx 0.15 \mathrm{~m}$ ). The prescribed upstream discharge is either taken in a Beta law or using the following relationships with $q_{\text {up }} \in[2 ; 4] \mathrm{m}^{2} \mathrm{~s}^{-1}$ (yielding a standard deviation of $\sigma_{q} \approx 0.27 \mathrm{~m}^{2} \mathrm{~s}^{-1}$ ). Three cases are studied, with different correlation values between the two parameters:

$$
R=\frac{\operatorname{cov}\left(h_{\mathrm{ds}}, q\right)}{\sigma_{h_{\mathrm{ds}}} \sigma_{q}}
$$

1. In the first cases, the parameters are assumed totally independent $(R=0)$.

2. In the second case, a total correlation $(R=1)$ is set between the parameters using the following stagedischarge relationship:

$$
q=\frac{1}{3} \sqrt{g\left(h_{\mathrm{ds}}-0.5\right)^{3}}
$$

this distribution being re-centred on $q^{0}$ (see table 1). 


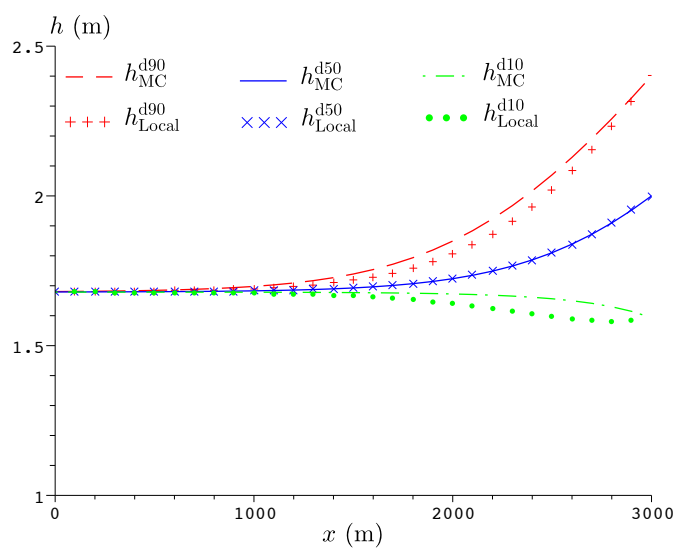

(a) $\psi=h_{d s}$

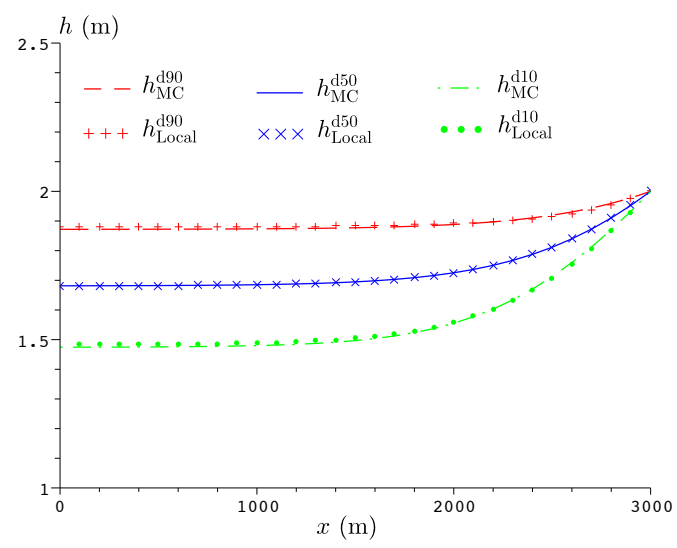

(c) $\psi=q_{u p}$

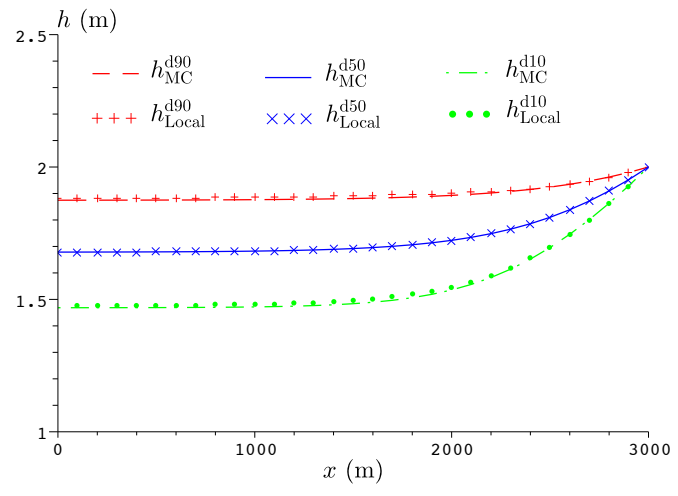

(b) $\psi=n_{M}$

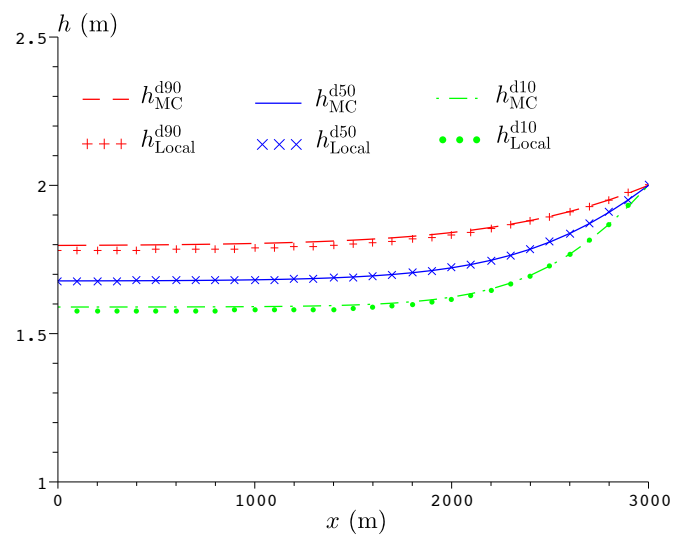

(d) $\psi=S_{0}$

Figure 2: Test 1a - OAT analysis. Deciles obtained from the MC simulations and retrieved using local sensitivity and Eq. (11)

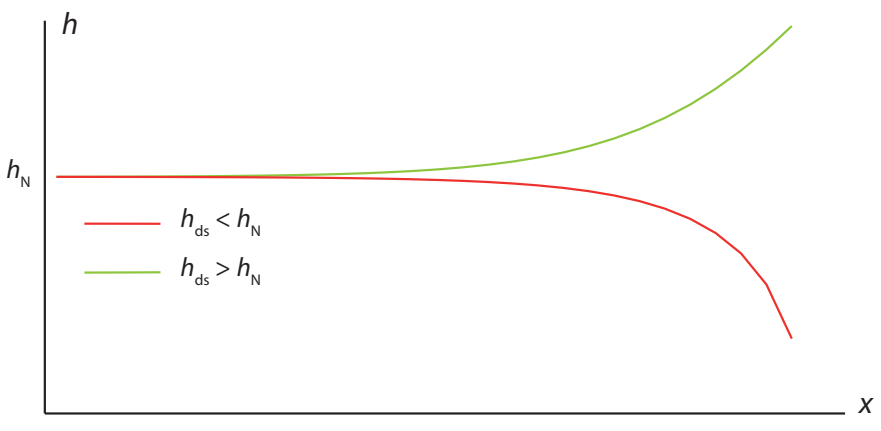

Figure 3: Different patterns of the flow solution depending on the prescribed downstream water depth 

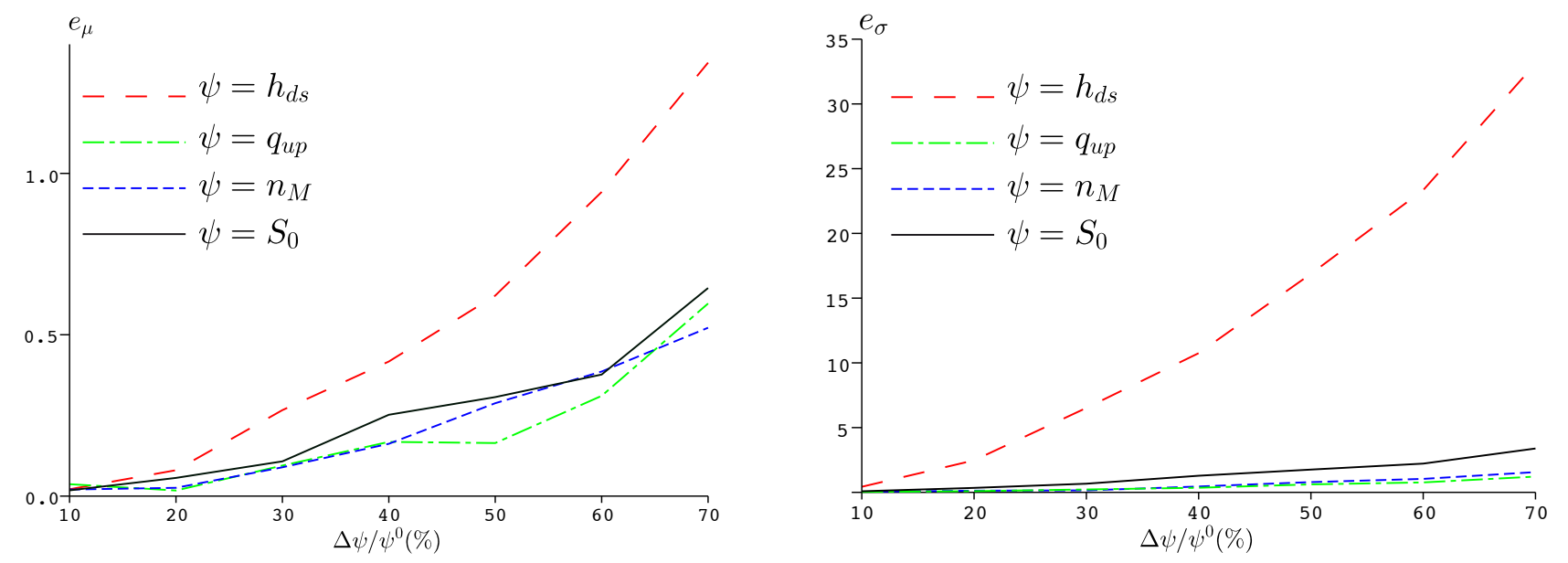

Figure 4: Test 1a - OAT analysis. Relative errors (Eqs. 8-9) of local estimates compared to global ones. Left: mean estimates, right: standard deviation estimates.

3. In the third case, a gaussian noise is added to equation (13) yielding a correlation $R=0.9$.

Figure 5 shows the standard deviation of the output variable $h$ obtained from Monte Carlo simulations and estimated from local method by Eq. (7), for the three different correlations between the two parameters. The relative differences between standard deviation estimates (Eq. (9)) is respectively $0.65 \%$ of global result for $R=0,0.2 \%$ for $R=0.9$ and $0.12 \%$ for $R=1$. This test case shows that the local sensitivity can be successfully used to reproduced uncertainty analysis of a global MC approach even when parameters are correlated.

\subsubsection{Simultaneous variation of four parameters}

In this section, we study the simultaneous variation of the four parameters, $h_{\mathrm{ds}}, n, q$ and $S_{0}$, considered as independent. To allow a computation in a reasonable time, the Latin Hyper-Cube sampling method is used: the probability density function of the Beta law is divided into 20 intervals of equal probability and one value is selected at random from each interval (see e.g. [Saltelli et al., 2000] for more details on this sampling method). This leads to a total number of simulations equals to $20^{4}=160000$ (in comparison to 5 simulations required for the local method). The four parameters are distributed along a Beta law, centred on the nominal values given in Table 1 and with $X \%$ simultaneous variation, with $X=20 \%, 30 \%$ or $40 \%$.

As in the previous section, the standard deviation of the results given by the Latin Hyper-Cube simulations is compared to its estimate from the local sensitivity and the variance of each parameter using equation (7) in Figure 6. The relative difference (Eq. 9) between both methods remains lower than $2.8 \%$ even when the variation of each parameter reaches as high as $40 \%$.

\subsection{Sudden opening of a gate}

The instantaneous opening of a gate in an irrigation channel can be represented using the classical dam-break problem [Stoker, 1957], that has been widely used as a validation test case for Shallow Water Equations solvers. This is an initial value problem, with the following initial conditions:

$$
\begin{aligned}
& h(x, 0)= \begin{cases}h_{L} \text { for } & x<x_{0} \\
h_{R} \text { for } & x>x_{0}\end{cases} \\
& q(x, 0)=0
\end{aligned}
$$

where $h_{L}$ is the water depth in the left-hand side of the gate and $h_{R}$ in the right-hand side, taken as zero in the following. The parameters of the test case are given in Table (2). At $t=0$, the instantaneous, total opening of the 

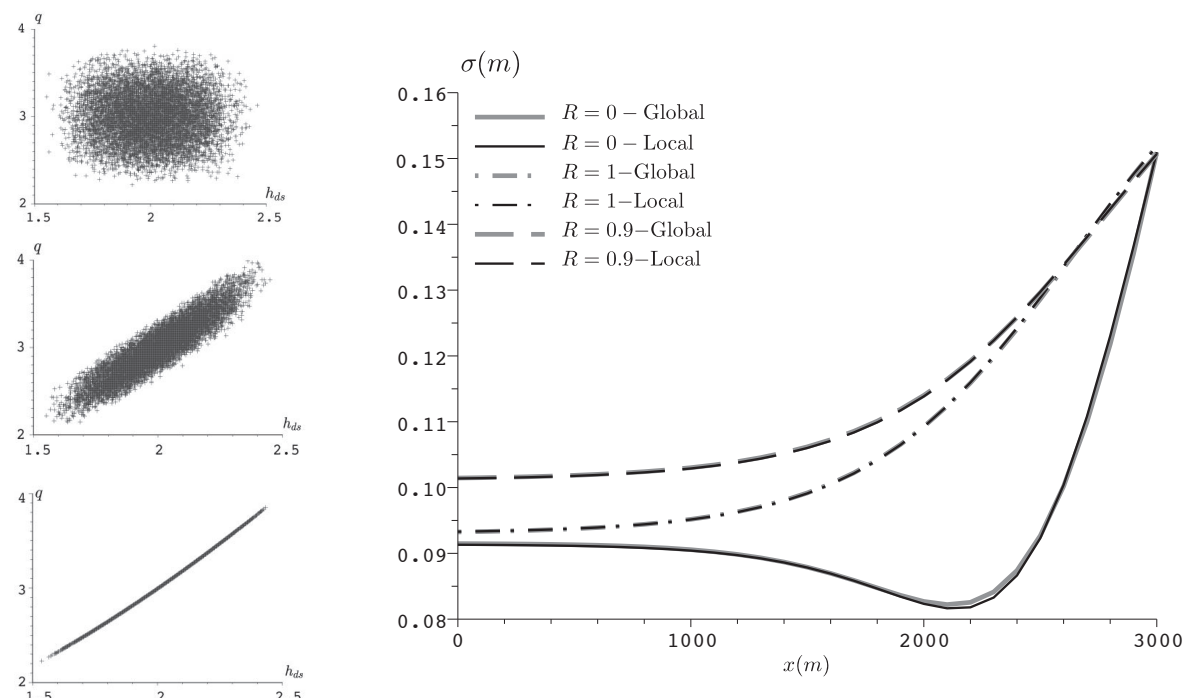

Figure 5: Test $1 \mathrm{~b}-$ two correlated parameters. Variance of the variable $h$ computed from Monte Carlo simulations and from local method for different values of the correlation $R$ (Eq. (12)) between the two parameters (left: distributions of the two parameters for $R=0,0.9$ and 1 ).

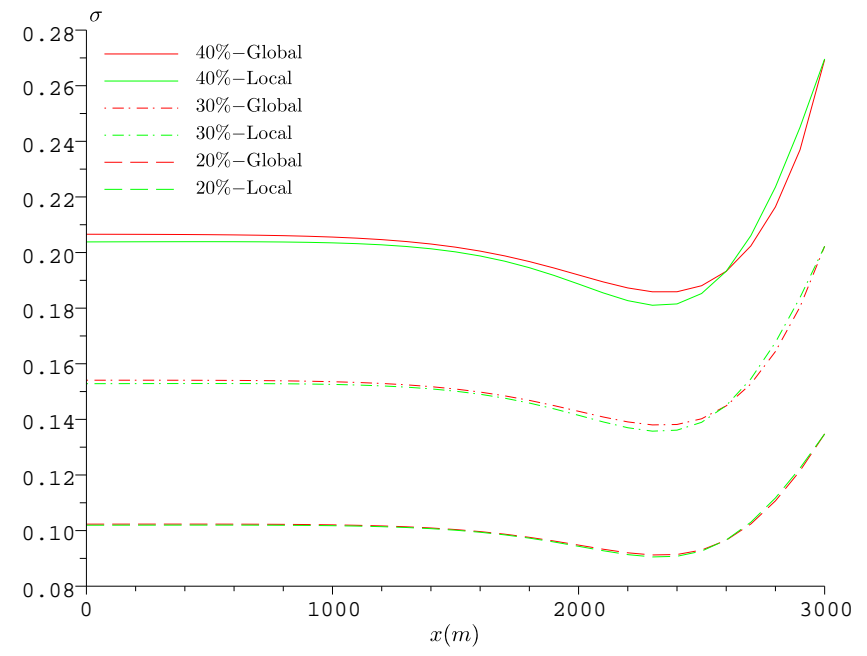

Figure 6: Test 1c - four uncertain parameters. Output standard deviation computed from Latin Hyper-Cube simulations and from local method for a simultaneous variation of the four independent parameters. Each parameter can vary from the mean value up to $X \%$ with $X=20,30,40$. The relative differences between both methods are equal to $e_{20 \%}=0.8 \%, e_{30 \%}=1.7 \%$ and $e_{40 \%}=2.8 \%$ of the global results. 


\begin{tabular}{lll} 
Symbol & Meaning & Value \\
\hline$g$ & Gravitational acceleration & $9.81 \mathrm{~m} \mathrm{~s}^{-2}$ \\
$L$ & Channel length & $200 \mathrm{~m}$ \\
$S_{0}$ & Bottom slope & $1 \cdot 10^{-3}$ \\
$n_{M}$ & Manning's friction coefficient & $0.02 \mathrm{~m}^{-1 / 3} \mathrm{~s}$ \\
$q$ & Prescribed unit discharge & $0 \mathrm{~m}^{2} \mathrm{~s}^{-1}$ \\
$h_{R}$ & Initial water depth in the right-hand side of the gate & $0 \mathrm{~m}$ \\
$h_{L}^{0}$ & Nominal value of the initial water depth in the left-hand side of the gate & $2 \mathrm{~m}$ \\
$\Delta x$ & Discretization cells width & $0.1 \mathrm{~m}$ \\
$T_{\max }$ & Simulation time & $6 \mathrm{~s}$ \\
\hline
\end{tabular}

Table 2: Test 2 - sudden opening of a gate. Parameters of the test case.
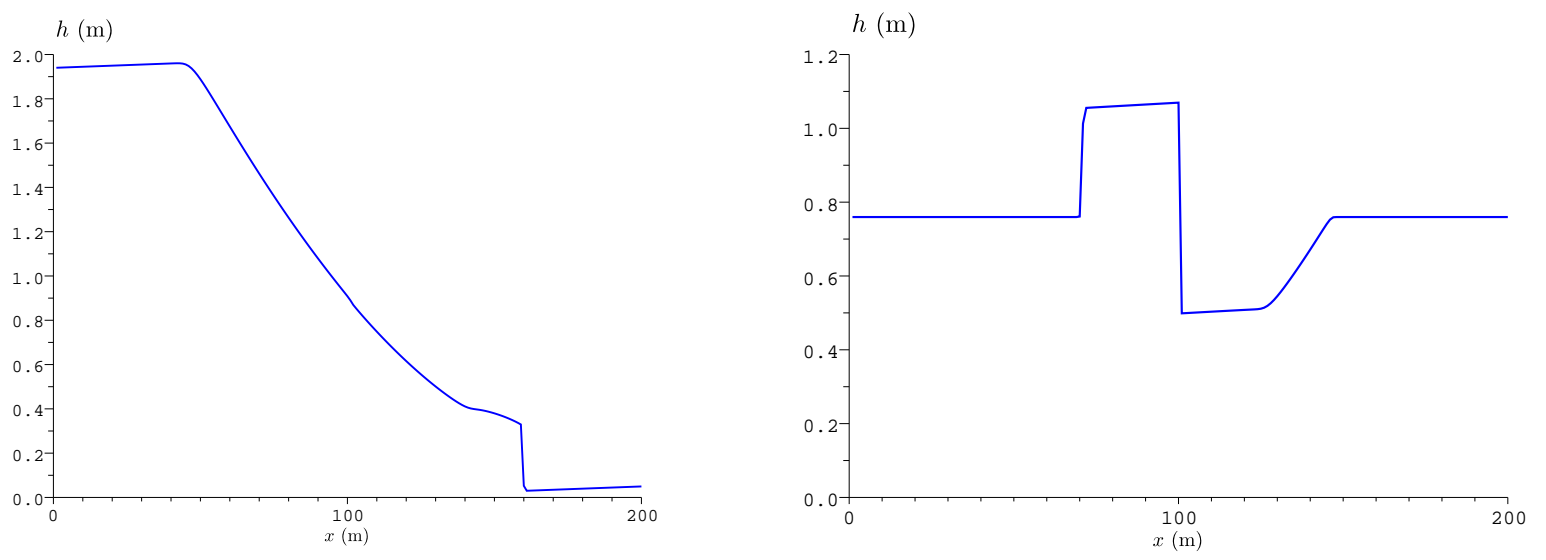

Figure 7: Test cases 2 and 3. Profile of the output water depth as a function of space $6 \mathrm{~s}$ after the instantaneous opening (left) and closing (right) of a gate.

gate suddenly releases the water that starts flowing from the left to the right. The output water depth obtained with the nominal parameters is plotted in Figure 7(left).

The uncertainty analysis is performed with respect to the initial water depth in the left-hand side of the gate $h_{L}$, taken in a Beta law with a possible variation of $\pm 30 \%$. We can see in Figure (8) a proper reproduction of the global standard deviation output (water depth and unit discharge) using the local sensitivity, over the whole domain except for the narrow shock area where the spike is sharper with the local approach. More results on this test case can be found in Delenne et al. [2012] where it is shown that uncertainty is properly estimated by the local method over the whole domain but the immediate vicinity of the shock even when the four parameters of the test case are allowed to vary simultaneously from their nominal values by $\pm 40 \%$.

\subsection{Sudden closing of a gate}

In this test case, the flow is initialy steady with a constant flow velocity and a water level assigned to a given value $h_{0}$. At $t=0$, a gate is instantaneously closed at the middle of the channel (see Figure 9). The profile of the output water depth obtained from the nominal values of the parameters (given in Table 3 ) is plotted in Figure 7(right). It can be seen that the gate closing induces a water level increase upstream to the gate. This may cause water overflow or damages in the channel. The most important parameter in this test case is the initial flow velocity. The uncertainty analysis is thus performed with respect to the initial flow velocity, taken from a Beta law with a possible variation of $\pm 50 \%$.

As in the previous test cases, the variances of the Monte Carlo simulations results (water depth and unit dis- 

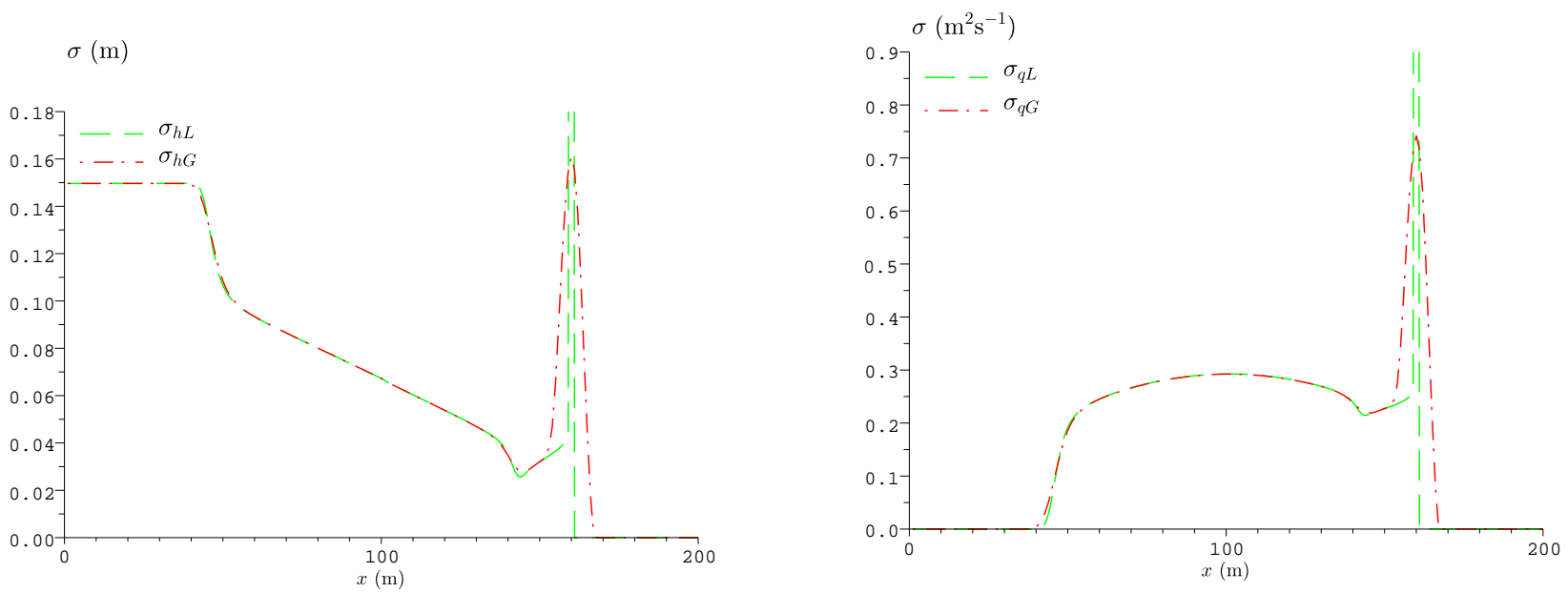

Figure 8: Test 2 - sudden opening of a gate. Output standard deviations estimated by local and global methods when the initial water depth in the left-hand side of the gate can vary by $\pm 30 \%$ in a Beta law. Left: output water depth standard deviation; right: output unit discharge standard deviation.
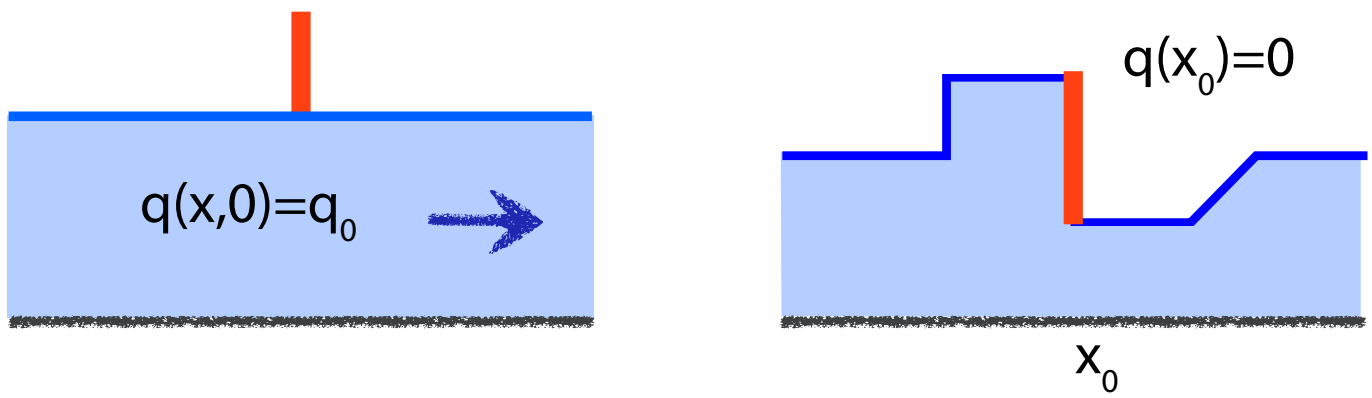

Figure 9: Test 3 - sudden closing of a gate. Definition sketch of the test case. 


\begin{tabular}{lll} 
Symbol & Meaning & Value \\
\hline$g$ & Gravitational acceleration & $9.81 \mathrm{~m} \mathrm{~s}^{-2}$ \\
$L$ & Channel length & $200 \mathrm{~m}$ \\
$S_{0}$ & Bottom slope & $1 \cdot 10^{-3}$ \\
$n_{M}$ & Friction coefficient & $0.02 \mathrm{~m}^{-1 / 3} \mathrm{~s}$ \\
$u^{0}$ & Nominal value of the initial and prescribed flow velocity & $1 \mathrm{~m} \mathrm{~s}^{-1}$ \\
$h_{0}$ & Initial water depth in the channel & $0.76 \mathrm{~m}$ \\
$\Delta x$ & Discretization cells width & $0.1 \mathrm{~m}$ \\
$T_{\max }$ & Simulation time & $6 \mathrm{~s}$ \\
\hline
\end{tabular}

Table 3: Test 3 - sudden closing of a gate. Parameters of the test case.
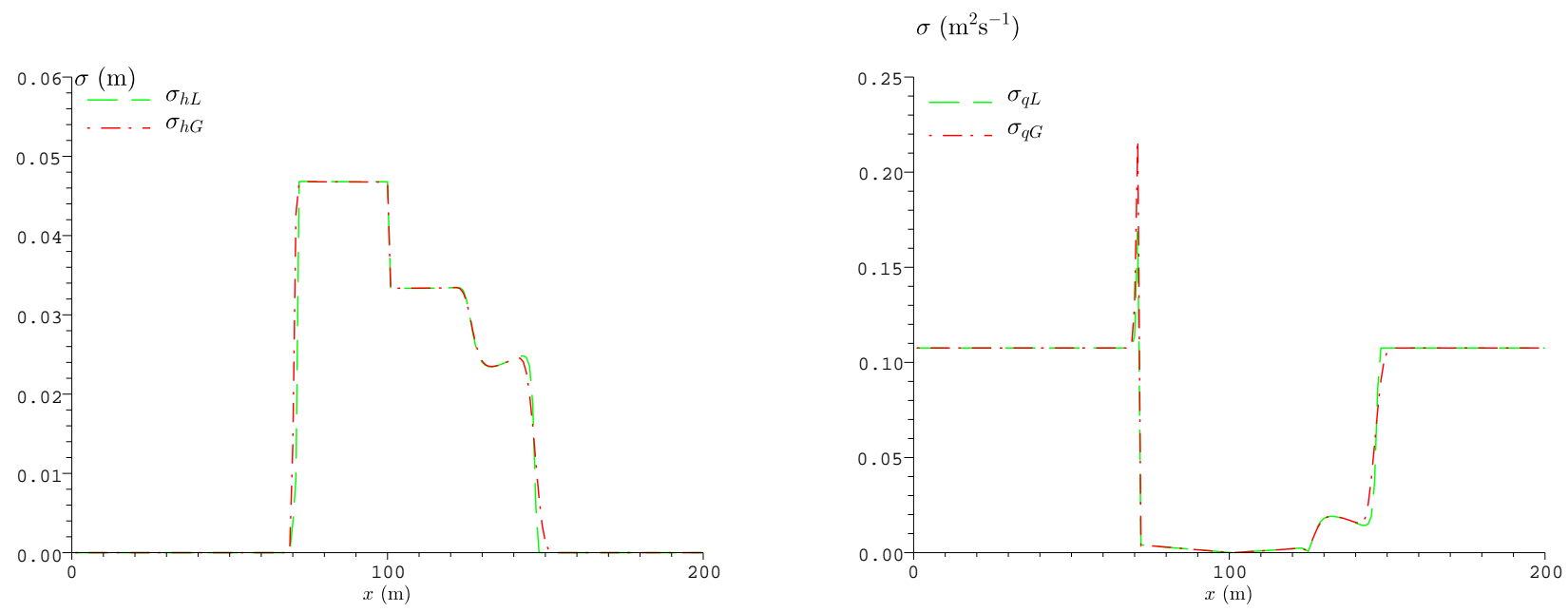

Figure 10: Test 3 - sudden closing of a gate. Output standard deviations estimated by local and global methods when the initial flow velocity in the channel can vary by $\pm 50 \%$ in a Beta law. Left: output water depth standard deviation; right: output unit discharge standard deviation.

charge) are compared to the estimations from local sensitivity (see Figure 10). Once again, the two approaches provides very close results even despite the discontinuous nature of the flow solution. The local approach thus enables, in two runs of the model, an accurate reproduction of results obtained with a high number of Monte Carlo simulations.

\section{CONCLUSION}

This study shows that the local sensitivity can be succesfully used in place of a global uncertainty analysis even for non linear equations such as the shallow water equations with discontinuous solutions. This yields considerable gain in computation time as the local sensitivity requires only $1+p$ simulations for $p$ parameters. Considering specific cases such as uncertainty analysis with respect to the downstream water depth for the backwater curve, known as critically non linear, an alternative would be to perform a second-order analysis, that would be more informative than the first-order analysis presented in this study and that requires only $1+2 p$ simulations.

Further works are devoted to more highly non linear equations, such as the Saint-Venant equations in a channel with high reach slope variations. In this case, the sensitivities of the model outputs to the state variables $A$ (crosssection area) and $Q$ (discharge), are expected to be more dependent on the nominal values chosen. A way to evade this issue may be to use smoother variables such as $u$ (flow velocity) or $c$ (wave celerity) in the sensitivity analysis, 
in place of the state variables.

Performing several local sensitivities could also give usefull information on the domains of the parameter space on which the model is more sensitive, while maintaining a limited number of simulations in comparison to the global approach.

\section{REFERENCES}

Beven, K. and Freer, J. (2001). Equifinality, data assimilation, and uncertainty estimation in mechanistic modelling of complex environmental systems using the GLUE methodology. Journal of Hydrology, 249(1-4):11-29.

Delenne, C., Cappelaere, B., and Guinot, V. (2012). Uncertainty analysis of river flooding and dam failure risks using local sensitivity computations. Reliability Engineering and System Safety, 107:171-183.

Delenne, C., Finaud-Guyot, P., Guinot, V., and Cappelaere, B. (2011). Sensitivity of the 1D shallow water equations with source terms: Solution method for discontinuous flows. International Journal for Numerical Methods in Fluids, 67:981-1003.

Delenne, C., Guinot, V., and Cappelaere, B. (2008). Direct sensitivity computation for the Saint-Venant equations with hydraulic jumps. Comptes Rendus Mécanique, 336:766-771.

Guinot, V., Cappelaere, B., and Delenne, C. (2009a). Finite volume solution of the one-dimensional shallow water sensitivity equations. Journal of Hydraulic Research, 47(6):811-819.

Guinot, V. and Delenne, C. (2012). MUSCL schemes for the shallow water sensitivity equations with passive scalar transport. Computers and Fluids, 59:11-30.

Guinot, V., Delenne, C., and Cappelaere, B. (2009b). An approximate-state Riemann solver for sensitivity equations with discontinuous solutions. Advances in Water Resources, 32(1):61-77.

Hall, J., Tarantola, S., Bates, P., and Horritt, M. (2005). Distributed sensitivity analysis of flood inundation model calibration. Journal of Hydraulic Engineering-ASCE, 131(2):117-126.

Harten, A., Lax, P., and Van Leer, B. (1983). On upstream differencing and Godunov-type schemes for hyperbolic conservation laws. Journal of Computational Physics, 50:235-269.

Kay, A. L. and Davies, H. N. (2008). Calculating potential evaporation from climate model data: A source of uncertainty for hydrological climate change impacts. Journal of Hydrology, 358(3-4):221-239.

Malaterre, P.-O., Baume, J.-P., Jean-Baptiste, N., and Sau, J. (2011). Analysis for the calibration of open-channel flow models: insights from linear systems and automatic control approaches. Houille Blanche - Revue Internationale de l'Eau, 3:63-68.

Minville, M., Brissette, F., and Leconte, R. (2008). Uncertainty of the impact of climate change on the hydrology of a nordic watershed. Journal of Hydrology, 358(1-2):70-83.

Pappenberger, F., Matgen, P., Beven, K. J., Henry, J.-B., Pfister, L., and De Fraipont, P. (2006). Influence of uncertain boundary conditions and model structure on flood inundation predictions. Advances in Water Resources, 29(10):1430-1449.

Roux, H. and Dartus, D. (2008). Sensitivity analysis and predictive uncertainty using inundation observations for parameter estimation in open-channel inverse problem. Journal of Hydraulic Engineering-ASCE, 134(5):541549. 
Saltelli, A., Chan, K., and Scott, E. (2000). Sensitivity analysis. Probability and Statistics series. John Wiley \& Sons publishers.

Stoker, J. J. (1957). Water Waves. Interscience, Wiley, New-York.

Toro, E., Spruce, M., and Speares, W. (1994). Restoration of the contact surface in the HLL-Riemann solver. Shock Waves, 4:25-34. 